\title{
Quel paradigme pour interpréter les genres télévisuels?
}

\section{François Jost}

Docteur; Sorbonne Nouvelle Paris III

francoisjost@orange.fr

\section{Résumé}

Les genres télévisuels sont nombreux et ils ne cessent de proliférer. Comment trouver les interprétants fondamentaux qui permettent de penser les genres passés et à venir? C'est à cette question que j'ai tenté de répondre à travers un modèle conceptuel qui a évolué au cours des ans, passant notamment d'une catégorisation en mode d'énonciation à une catégorisation en monde. De mode à monde, il y a un changement de paradigme. Cet article propose donc un retour épistémologique sur trois façons d'envisager les genres télévisuels. D’abord comme une promesse qui guide les inférences et les croyances des téléspectateurs, et qui peut être identifiée à un niveau sémiologique, ensuite, à un niveau pragmatique, en fonction de la reconnaissance d'un mode d'énonciation qui conditionne la valeur de vérité du programme, enfin par l'ancrage dans un monde, modèle qui permet d'expliquer pourquoi l'interprétation des genres télévisuels est un enjeu juridique, économique et communicationnel.

\section{Mots clefs}

Télévision. Genres télévisuels. Interprétation. Enonciation. Promesse. Mondes.

\section{Introduction}

Très régulièrement, des étudiants viennent me voir et me demandent à quel genre appartient telle ou telle émission qui vient d'être diffusée sur le petit écran. Et, à chaque fois, c'est le même embarras: comment leur répondre? Comment leur dire que ce n'est pas moi, pas plus que je ne sais quel autre expert, qui décide du genre d'un programme, et, en même temps, qu'on ne peut faire confiance à n'importe quelle étiquette générique donnée par le producteur ou le diffuseur? 
Cet embarras, à y réfléchir, me semble tenir à une ambiguïté qui touche toute réflexion sur les genres: d'un côté, l'attribution d'une étiquette générique à un texte, quel qu'il soit, verbal ou audiovisuel, est une opération relativement arbitraire: un poème de Ronsard peut aussi bien être classé dans une anthologie poétique, que dans un recueil des plus belles déclaration d'amour ("Mignonne, allons voir si la rose...") ou que dans un traité de versification. De même, toute qualification générique peut donner lieu à une régression à l'infini: un "roman"peut être un "roman réaliste", voire un "roman balzacien", etc. D’un autre côté, nous avons la conviction que l'extension de tel ou tel genre n'est pas indéfinie, qu'il faut lui imposer des limites et, dans ces conditions, que toutes les attributions génériques à un objet ne sont pas également acceptables.

Une seule chose est sûre: c'est que, pour regarder un document, pour lire un livre, nous avons besoin de ramener l'inconnu au connu, ce qui est le propre de l'étiquetage générique. Quelle part faire à notre connaissance des genres a priori comme condition transcendantale de l'interprétation, comme horizon d'attente d'un texte? Quelles sont les lignes de critères qui nous permettent de catégoriser les genres? Et dans quelle mesure dépendent-ils du modèle de la communication qui les pense? Voici trois questions auxquelles j'essaierai de répondre en montrant que ces réponses engagent des paradigmes différents.

Par quel bout prendre le genre? Une façon de se débarrasser de cette hésitation, est de décider que l'on ne va s'intéresser qu'aux étiquettes génériques. C'est le parti pris de Schaeffer dans Qu'est-ce qu'un genre littéraire? (1989). Sa démarche est séduisante et, je dois bien l'avouer, elle est partiellement à la base de mon interrogation sur les genres télévisuels.

Pourtant, si elle met en valeur le fait, particulièrement vrai pour la télévision, que les noms de genres renvoient à des réalités communicationnelles bien différentes, son approche a, pour moi, deux défauts: elle donne un rôle exagéré à l'onomaturge, qu'il soit producteur ou diffuseur, et elle aboutit à l'inverse de ce à quoi elle voulait parvenir: tout en voulant adopter une perspective communicationnelle, elle fige les genres en une répartition statique qui serait valable pour n'importe qui, quel que soit l'usage que l'on fait du genre.

Comment donc considérer le genre par en tenant compte à la fois de l'intentio auctoris, de l'intentio operis et de l'intention lectoris, selon la tripartition proposée par Eco? Voici l'interrogation à laquelle je tente de répondre depuis une quinzaine d'années. Au fil des ans, j'ai pourtant opéré un déplacement terminologique sans prendre le temps de m'interroger sur ce qu'il signifiait. 
Après avoir catégorisé les genres en fonction de leur mode d'énonciation, j'en suis venu, quelques années plus tard, à les catégoriser en fonction de leur appartenance à un monde. De mode à monde, il n'y a qu'une lettre de différence, mais je sens confusément que c'est bien plus: plutôt un changement de paradigme qu'une faute de frappe! Ou, peut-être, cette hésitation que je viens d'évoquer. J'aimerais, dans cet article, adopter une démarche épistémologique et m'interroger sur ce glissement pour comprendre ce que l'on gagne ou ce que l'on perd à adopter l'un ou l'autre de ces paradigmes.

\section{Ontologie du genre: connaissance des genres}

À l'origine de ma réflexion sur le genre, il y a d'abord, la décision théorique de le considérer, non comme une catégorie stable et intangible, ayant une signification en soi, mais en tant que réalité communicationnelle, à l'instar de J.-M. Schaeffer (1989). Ce choix m'apparut, et m'apparaît encore, comme nécessaire pour réagir aux impasses d'une sémiologie immanentiste qui faisait de la production du sens une opération uniquement textuelle. Dans la mesure où le genre est une catégorie nécessaire à l'interprétation, le premier mouvement est de l'envisager depuis le spectateur, si je puis dire. Dans cette perspective, les travaux de Sperber et Wilson (1989), qui s'en prenaient vivement à la notion de code dans La Pertinence, laissaient entrevoir des avancées fructueuses, en mettant en lumière que la communication verbale était largement fondée sur des inférences des acteurs et sur leur partage d'un univers cognitif commun.

La fonction cognitive du genre, pour le téléspectateur, pouvant être définie de façon générale comme une recherche de pertinence, c'est-à-dire comme un effort pour augmenter sa connaissance du monde, il est naturel de se demander, dans un premier temps, comment le spectateur fait pour ramener l'inconnu au connu, en d'autres termes, quelles inférences il met en œuvre à partir de la réalité textuelle. Je fais donc l'hypothèse, dans Un monde à notre image (JOST, 1992), puis dans Le Temps d'un regard (JOST, 1998a), que l'interprétation des documents dépend des inférences associées a priori aux différents genres. Celles-ci sont comme un cadre a priori de l'intelligibilité du document. Si je choisis d'aller voir au cinéma une comédie plutôt qu'un film policier, c'est parce que j'ai envie de rire et que je m'attends à rire. Si les téléspectateurs aiment le direct, c'est parce qu'il semble moins manipulé qu'un programme en différé monté après coup. Mais tout n'est pas toujours si simple et limpide. C'est en effet en ce point que le bât blesse. Si les attentes génériques sont indubitables 
s'agissant des deux exemples que je viens de donner, les théoriciens de la littérature, du cinéma et de la télévision considèrent en général que la fiction fait l'objet d'un consensus qui se résume en une formule bien connue, celle de Coleridge, "the willing suspension of disbelief". Or, cette formule est bien plus une prescription qu'une description d'un processus empirique.

J'ai souvent cité cette observation d'Eco qui, au moment où il devient un romancier à succès, s'aperçoit que le fameux contrat de fiction qu'il a soutenu auparavant n'est pas partagé par ses lecteurs:

Ayant fait moi-même l'expérience d'avoir écrit deux romans qui ont touché des millions de lecteurs, je me suis rendu compte d'un phénomène extraordinaire. Jusqu'à quelques dizaines de milliers d'exemplaires (estimation variable d'un pays à l'autre), on touche en général un public connaissant parfaitement le pacte fictionnel. Après, et surtout au-delà du million d'exemplaires, on entre dans un no man's land où il n'est pas sûr que les lecteurs soient au courant de ce pacte. (ECO, 1996, p. 102)

$\mathrm{Si}$, dès que l'on passe au million de lecteurs, ce " pacte " n'est plus connu, que dire de la télévision française qui implique, chaque jour, au moins deux dizaines de millions de téléspectateurs?

Mais il n'est pas nécessaire d'invoquer ce seuil du million de téléspectateurs pour mettre en doute la fameuse "willing suspension of disbelief". Qui d'entre nous n'a pas fait l'expérience, en visionnant un film ou une série, de sortir de cette attitude en pensant dans son for intérieur ou non, face à une séquence par trop tirée par les cheveux, "là, ils exagèrent!", " ça n'est pas possible! ". En bref, " je n'y crois pas. ”

Le principal inconvénient du contrat de fiction est son aspect massif. Comme si la croyance était une, unitaire et monolithique. Or, la suspension de l'incrédulité est un phénomène beaucoup plus subtil et plus polymorphe que le laisse accroire ce modèle, qui suppose qu'on donne un blanc-seing à l'auteur. Une chose est la présence de postulats, qui rendent cohérent l'ensemble du film (une radiation atomique fait rapetisser), une autre chose est de considérer que tout dans le film se rabat sur quelques postulats.

Je peux croire à une explication d'ensemble, mais pas aux détails ou l'inverse.

La croyance à la fiction dépend aussi de ce que je veux croire et, donc, de la question de savoir si j'ai de la sympathie ou non pour la fiction que l'on me construit. Par exemple, je regarde la série américaine $O z$, qui décrit un univers carcéral que je trouve extrêmement convainquant. Un téléphone portable a été introduit en cachette par un nouveau venu. Au bout de quelques épisodes je me demande comment ce téléphone marche toujours, alors 
qu'il n'a pas de recharge pour la batterie, mais, finalement, je m'en fiche car ce que cela provoque comme sentiments entre les personnages et comme logique d'action me plaît. Autre exemple: le personnage qui creuse son tunnel, sans qu'on se demande vraiment où est mise la terre. Pris par l'action, j'oublie de me poser la question.

A d'autres moments, ma crédulité grippe. Regardant le film de J. J. Abrams, Super 8, qui raconte comment le déraillement d'un mystérieux train de marchandises va libérer des matières étranges et bientôt une bête extraterrestre. Le film réemprunte tous les chemins stéréotypés d'Hollywood (le monstre qui cède au petit garçon comme King Kong à la bête). Le groupe d'enfants héros de cette histoire regarde des films qu'ils trouvent dans une boîte et qui vont expliquer le mystère de la bête. Ces film ont le son: comment? Je n'ai pas envie de faire grâce de ce détail à l'auteur. Parce que le film repose sur trop de conventions. Je refuse de suspendre mon incrédulité.

On ne se donne pas à un film ou à un livre parce qu'il y a seulement marqué "fiction " et qu'il le demande. Il faut en avoir envie, il faut donc que la fiction produise une certaine sympathie en nous, qui va dicter ou non notre indulgence et notre soif de savoir. La " suspension of disbelief" n'est qu'une attitude vertueuse que le narratologue recommande aux autres, mais qu'il est incapable de respecter. Prenons-la, donc, pour ce qu'elle est: une maxime à l'usage des enfants qui doivent pas croire tout ce qu'ils voient !

Plutôt que de recourir à la métaphore du contrat, qui suppose résolu ce qu'il faut justement expliquer et démontrer, j'ai proposé de dire, dans un premier temps que, pour qui sait ce que sont les genres, il découle de ce savoir des inférences sur l'utilisation des codes audiovisuelles. Inférences qui sont résumées dans ce tableau des inférences spectatorielles que j'ai proposé dans Introduction à l'analyse de la télévision, en 1999, et qui doit donc être relu à la lumière de ce que je viens de dire comme les inférences qui doivent se produire dans la tête de ceux qui ont des connaissances sur ce que sont et engagent l'enregistrement automatique, le direct préparé et non préparé, le direct, le documentaire, la fiction ou l'œuvre d'art. Savoirs qui sont apportés par la sémiologie ou la narratologie (voir page 6). Tous ces savoirs devraient faire l'objet d'une leçon entrant dans un programme d'éducation aux médias. Bien sûr, ces savoirs peuvent aussi amener le téléspectateur à formuler, en cours de projection, une ou plusieurs hypothèses sur la nature du programme, notamment sur le degré d'intervention humaine dans l'élaboration du document et sur sa finalité.

A titre d'exemple, on peut observer les cases "direct non préparé" et "direct préparé " et les lire de la façon suivante: un direct non préparé, c'est-à-dire l'irruption d'un 
événement dans le champ des caméras qui, comme le 11 septembre, impose une mise en cadre peu intentionnelle, un ordre temporel subi; la succession, contrairement, par exemple, à la retransmission d'une cérémonie ou d'un match de football, qui peut signifier la simultanéité par la construction de plusieurs séries narratives (cf. l'enterrement de Mitterrand avec ces deux lieux, Paris et Jarnac) en vue de rendre lisible l'action. Le direct non préparé, parce qu'il diminue l'intentionnalité humaine, paraît plus authentique que le direct préparé, qui cherche à rendre lisible.

Remarque importante: ces savoirs, même quand on les possède, ne sont pas prescripteurs au point d'empêcher des téléspectateurs de regarder un direct comme une œuvre plus que pour son indicialité - comme on le voit parfois avec les amateurs de football, qui visionnent après coup un match victorieux de l'équipe de France (on se console comme on peut!). Rien n'empêche non plus de lire un sonnet de Ronsard comme un témoignage d'amour plus que comme un poème. En d'autres termes, le champ de l'interprétation n'épuise pas celui des usages.

Ce que montre le tableau suivant, en deuxième instance, c'est la corrélation des croyances et des savoirs avec des inférences comme: "si c'est en direct, cela doit être authentique".

En somme, cette approche du genre tente d'expliquer ce que l'idée de genre change à la réception d'un document et, dans cette mesure, elle est essentiellement attentionnelle. Elle pousse à l'extrême la conception du genre comme horizon d'attente. 
Table 1 - Types d'inférences en fonction du savoir sur les types de documents

\begin{tabular}{|c|c|c|c|c|c|c|c|c|}
\hline Type & \multicolumn{2}{|c|}{ Dispositio } & Inventio & Narrativité & Promesse & $\begin{array}{c}\text { Figure } \\
\text { anthropoïde }\end{array}$ & $\begin{array}{c}\text { Type } \\
\text { d'énonciation }\end{array}$ & $\begin{array}{l}\text { Intention } \\
\text {-nalité }\end{array}$ \\
\hline $\begin{array}{c}\text { Enregistrement } \\
\text { automatique }\end{array}$ & $\begin{array}{l}+\mathrm{ou}- \\
\text { aléatoire }\end{array}$ & non & non & en deçà & $\begin{array}{c}\text { Garantie } \\
\text { d'authen- } \\
\text { ticité }\end{array}$ & - & - & \\
\hline $\begin{array}{c}\text { Direct non } \\
\text { préparé }\end{array}$ & $\begin{array}{c}\text { mise en cadre } \\
+ \text { ou }- \\
\text { maîtrisée }\end{array}$ & subi & non & & $\begin{array}{l}\text { Promesse } \\
\text { d'authen- } \\
\text { ticité }\end{array}$ & témoin & $\begin{array}{l}\text { accent sur } \\
\text { énonciation } \\
\text { filmique }\end{array}$ & \\
\hline Direct préparé & $\begin{array}{c}\text { mise en cadre } \\
\text { et points de } \\
\text { vue }+ \text { ou }- \\
\text { maîtrisés }\end{array}$ & $\begin{array}{c}\text { Travail sur la } \\
\text { simultanéité } \\
\text { + ou - } \\
\text { possible }\end{array}$ & non & $+\mathrm{ou}-$ & $\begin{array}{c}\text { Promesse } \\
\text { de lisibilité } \\
\text { du réel } \\
\text { accrue }\end{array}$ & $\begin{array}{c}\text { Du témoin } \\
\text { au } \\
\text { narrateur }\end{array}$ & $\begin{array}{c}\text { énonciation } \\
\text { filmique + } \\
\text { énonciation } \\
\text { narrative - }\end{array}$ & \\
\hline Documentaire & $\begin{array}{c}\text { construction } \\
\text { de l'espace en } \\
\text { fonction du } \\
\text { réel }\end{array}$ & $\begin{array}{l}\text { construction } \\
\text { du temps en } \\
\text { fonction du } \\
\text { réel }\end{array}$ & non & & & & $\begin{array}{c}\text { énonciation } \\
\text { filmique } \\
+ \text { ou }- \\
\text { énonciation } \\
\text { narrative - }\end{array}$ & \\
\hline Fiction & $\begin{array}{l}\text { Construction } \\
\text { de l'espace } \\
\text { en fonction } \\
\text { de l'intrigue }\end{array}$ & $\begin{array}{l}\text { Construction } \\
\text { du temps } \\
\text { en fonction } \\
\text { de l'intrigue }\end{array}$ & oui & + & $\begin{array}{c}\text { Promesse } \\
\text { de } \\
\text { pertinence } \\
\text { narrative du } \\
\text { visible et de } \\
\text { l'audible }\end{array}$ & Narrateur & $\begin{array}{c}\text { énonciation } \\
\text { narrative + } \\
\text { énonciation } \\
\text { filmique - }\end{array}$ & \\
\hline $\begin{array}{c}\text { Film comme } \\
\text { œuvre }\end{array}$ & $\begin{array}{c}\text { Construction } \\
\text { de l'espace en } \\
\text { fonction } \\
\text { du beau }\end{array}$ & $\begin{array}{l}\text { Construction } \\
\text { du temps en } \\
\text { fonction } \\
\text { du beau }\end{array}$ & oui & au-delà & $\begin{array}{c}\text { Promesse } \\
\text { de } \\
\text { pertinence } \\
\text { artistique } \\
\text { du visible et } \\
\text { de l'audible }\end{array}$ & Artiste & $\begin{array}{c}\text { Enonciation } \\
\text { cinématog- } \\
\text { graphique }\end{array}$ & 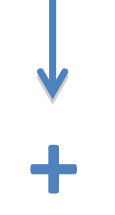 \\
\hline
\end{tabular}

Source: l'auteur.

\section{La reconnaissance du genre comme énonciation}

Le paradigme théorique précédent prend pour objet les genres les plus connus hérités du cinéma ou de la radio (et, en ce sens, il est marqué par mon parcours de chercheur). A cet égard, il ne rend pas compte de l'état de fait de la télévision de marketing, qui est marqué par une prolifération de nouveaux genres ou, tout au moins, de nouvelles étiquettes génériques. Néanmoins, il s'en dégage une grande ligne de partage entre les genres qui renvoient à la réalité et ceux qui renvoient à la fiction. De ce point de vue, on peut aussi tracer une ligne qui sépare les genres relevant de l'indicialité et ceux relevant de l'iconicité. L'enregistrement automatique ou la caméra cachée ressortissent à la première, alors que le documentaire introduit déjà une dose forte d'iconicité par la mise en forme intelligible du réel. Ces considérations n'ont aucune pertinence dans le paradigme narratologique 
genettien, qui avait été le mien jusqu'alors, dans la mesure où celui-ci boute l'auteur en dehors de son champ1. Ce qui n'est pas le cas, en revanche, de la narratologie de Käte Hamburger, dont la conception pragmatique de l'énonciation invite à faire éclater le carcan du texte. En considérant que la différence entre l'énoncé de réalité et l'énoncé de fiction n'est pas dans l'objet, mais dans le sujet qui le profère, elle opère un véritable retournement copernicien. Rappelons que, pour Käte Hamburger, la frontière entre réalité et fiction passe par le Je qui est à son origine. Dans la fiction qui, pour la théoricienne, ne s'exprime bien qu'à la troisième personne, les énoncés sont renvoyés à des Je-Origines fictifs, des personnages, d'où " l'expérience de non-réalité qui est au fondement de l'expérience psychologique du lecteur" (HAMBURGER, 1986, p. 75). L'usage de la première personne, en revanche, provoque inévitablement le "sentiment de vécu". D’où la difficulté à faire le départ entre roman à la première personne et une autobiographie: le premier a toujours un petit air d'auto-fiction qui l'ancre dans le réel.

Cette opposition ente le Je-origine réel et le Je-origine fictif permet de différencier des configurations audiovisuelles semblables à première vue, comme la voix over d'une série ou de celle d'un documentaire: quand bien même l'une et l'autre auraient exactement la même rhétorique, elles se distinguent toujours par le degré de réalité du narrateur. Sur ce point, je rejoindrai Eco, qui voyait aussi une séparation radicale entre réalité et fiction. Néanmoins, cette logique de partage n'est pas suffisante, elle laisse de côté de nombreux genres: les jeux, les divertissements, les variétés etc., ce dont le sémiologue italien a d'ailleurs conscience quand il se demande à propos du jeu: “ Dit-il la vérité ou bien met-il en scène une fiction?" (ECO, 1991, p. 203). Si l'on pousse un plus loin la réflexion, il apparaît clairement: 1) que tout jeu n'est pas fictif (par exemple, le saut à l'élastique ou un quiz ne sont pas fictifs) ; 2) que les fictions sont plus ou moins ludiques selon leur genre (le film politique moins que le jeu vidéo ou le film “post-moderne” [Jullier], tous fictifs, ne sont pas équivalents).

Comme son sait, Searle trace lui aussi la frontière entre énoncé de réalité et énoncé de fiction: le premier est une assertion littérale et sérieuse qui fait référence à notre monde, alors que le second est une assertion littérale, mais non sérieuse, qui feint de faire référence. Toutefois, le philosophe rajoute une troisième configuration, l'assertion sérieuse non littérale, qu'il appelle “figurale" et qui désigne les énoncés métaphoriques, comme, par

\footnotetext{
1 "Je ne suis pas sûr de rester dans les limites du champ narratologique en évoquant, au titre des questions de voix ("Qui par-
} le?"), le toujours épineux rapport entre narrateur et auteur " (GENETTE, 1991, p. 79). 
exemple, Hegel est un rossignol. Or, pour le philosophe des jeux Jacques Henriot, le jeu est précisément défini comme un procès métaphorique:

On appelle jeu tout procès métaphorique résultant de la décision prise et maintenue de mettre en œuvre un ensemble plus ou moins coordonné de schèmes, consciemment perçus comme aléatoires pour la réalisation d'un thème délibérément posé comme arbitraire. (HENRIOT, 1989, p. 300).

D’où ma proposition de classer les genres en fonction de trois modes d'énonciation: le mode authentifiant, qui regroupe les émissions tenant des assertions sérieuses et littérales sur le monde et qui relèvent, en dernière instance, d'un exercice de la preuve ; le mode fictif, qui regroupe les genres reposant sur la cohérence de l'univers créé; et, entre les deux, le mode ludique, "[...] où la réalité n'est pas tout à fait prise pour ce qu'elle est et où se constitue un monde qui obéit à ses propres règles." (c'est ma formulation de JOST, 1998b).

Illustration 1 - Les modes d'énonciation télévisuelle

\section{LES MODES D'ÉNONCIATION TÉLÉVISUELLE}

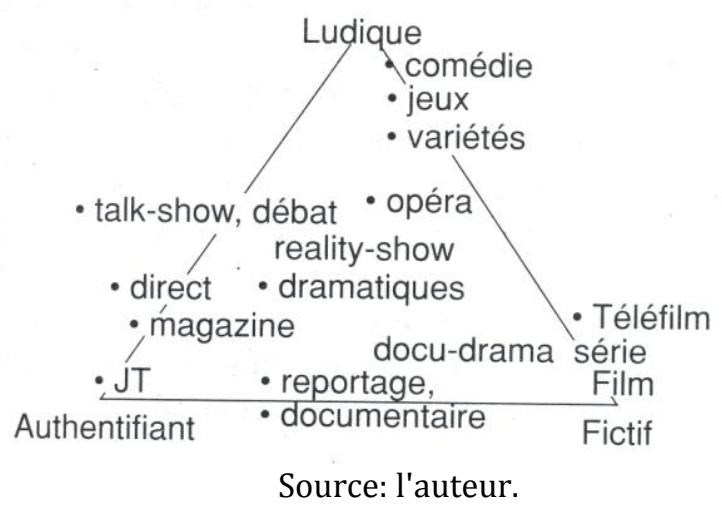

L'interprétation du genre dépend alors de la reconnaissance d'un mode d'énonciation qui le conditionne, et qui dépend très largement de l'identification du responsable de l'énonciation. Si je reprends l'exemple de la voix over, je peux proposer le tableau suivant qui différencie les responsables de l'énonciation: le journaliste, l'animateur et le personnage-narrateur: 
Table 2 - Types de médiations verbales

\begin{tabular}{|l|l|}
\hline $\begin{array}{l}\text { Responsable de } \\
\text { l'énonciation }\end{array}$ & $\begin{array}{l}\text { Rans une situation de commentaire } \\
\text { (over) }\end{array}$ \\
\hline $\begin{array}{l}\text { Je-origine réel (journaliste) } \\
\text { Je-origine ludique (animateur) }\end{array}$ & $\begin{array}{l}\text { - reportage: commentaire sur le } \\
\text { monde } \\
\text { Assertion sérieuse et littérale } \\
\text { Ressortit vrai vrai (conformité au } \\
\text { réel) }\end{array}$ \\
\hline $\begin{array}{l}\text { Je-origine fictif (narrateur } \\
\text { explicite) }\end{array}$ & $\begin{array}{l}\text { Vidéogag: commentaire sur } \\
\text { l'image }\end{array}$ \\
& $\begin{array}{l}\text { Assertion sérieuse non littérale } \\
\text { (conformité à la règle) }\end{array}$ \\
\hline & $\begin{array}{l}\text { vérieuse, mais littérale } \\
\text { Ressortit au vraisemblable }\end{array}$ \\
\hline
\end{tabular}

Source: l'auteur.

Alors que l'énoncé de réalité doit s'ajuster au réel, et que le locuteur doit en répondre et être capable de l'étayer par des preuves, l'énoncé ludique est soumis à des règles ou des rites qui dépassent le locuteur et qui relèvent généralement d'un jury chargé d'assurer le bon fonctionnement du jeu. Quant à l'objet de l'énoncé, il diffère lui aussi: la voix over du journaliste vise le monde, alors que celle de Videogag, par exemple, est d'abord un métacommentaire sur l'image.

L'avantage de ce modèle c'est qu'il permet d'articuler le niveau narratologique et la théorie des genres. En effet, quel que soit le genre, la responsabilité de l'énonciation verbale peut se décrire selon le même schéma structural, mais le statut logique des énoncés proférés est différent selon le mode d'énonciation qui leur est attribué.

En somme, ce modèle théorique permet de montrer que l'interprétation d'un programme est variable, et qu'elle dépend de l'attribution de ce programme à tel ou tel mode d'énonciation. Il permet aussi de circonscrire le terrain où s'opère la créativité générique. A une dérive continuelle d'un modèle en fonction de l'émergence de nouveaux genres, il oppose une sorte de catégorisation en archigenres qui permet d'expliquer l'interprétation. Néanmoins, deux événements de 2001 vont m'amener à le modifier. 


\section{Pragmatique du genre: croyance au genre}

Le premier est un événement aux répercussions mondiales, l'attentat du World Trade Center ; le second, un événement national, dérisoire en regard du premier, mais qui fut assez considérable pour être par trois fois à la Une du quotidien français Le Monde: l'arrivée en France d'une adaptation de Big Brother.

Or ces deux événements, considérés sous l'angle sémiotique, interrogeaient, chacun à sa manière, la question de la catégorisation des images et la problématique du genre.

"Breaking news" surgies brutalement dans le flot télévisuel dont elles arrêtèrent le cours, les images du 11 septembre posèrent un problème d'interprétation à de nombreux téléspectateurs. Une de mes connaissances, professeur de philosophie, crut d'abord qu'il s'agissait d'un film catastrophe, puis, changeant de chaînes et retombant sur les mêmes images, il crut à la programmation d'un film unique en raison d'une grève du service public, avant de comprendre qu'il s'agissait d'un événement en train de se passer dans le vrai New York. Cette réception particulière attira mon attention sur deux faits.

Le premier, est que la reconnaissance dépend bien d'un savoir (on ne reconnaît que ce qu'on connaît, disait en substance Platon) et qu'en l'absence de ce savoir, le sens des images dépend globalement d'un interprétant, au sens peircien, qui, écrase tout autre différence sémiologique (car, pour celui qui possède le savoir des genres, les images de destruction de la pointe de Manhattan différaient de celles d'Independance Day par le manque de raccords de regard qui construisent ou révèlent un Je-origine fictif). En l'occurrence, les deux interprétants mobilisés dans la réception de cet événement étaient le monde réel et le monde fictif.

Le second événement médiatique, l'apparition de Loft Story, invitait à poursuivre dans cette voie. Voilà un programme qui se présentait comme une "télé-réalité", se fondant sur un argument fondamental, à savoir que "rien n'échappera[it] aux 26 caméras " filmant en direct onze candidats à qui il était seulement été demandé d'être eux-mêmes. Dès lors, la question de la responsabilité de l'énonciation devenait problématique: s'agissait-il d'une captation automatique, purement indicielle, ou d'une captation intentionnelle? Les candidats étaient-ils les auteurs de leurs répliques ou, au contraire, les marionnettes de narrateurs tout puissants, tapis, si ce n'est dans l'ombre, au moins à l'abri d'une régie? Dans le monde entier se posaient les mêmes questions: ces jeunes captifs étaient-ils sincères ou jouaient-ils dans un récit organisé par d'autres? La suite de la télé-réalité donnera encore un peu plus de grain à moudre à ces interrogations, au point que savoir si un candidat était un 
je-réel ou un je-fictif devint l'objet de multiples actions en justice. La plus célèbre fut celle qui toucha l'Ile de la tentation.

La promesse pragmatique situait clairement le programme du côté de la réalité, en se présentant comme une expérience de mise à l'épreuve: “Durant 12 jours et 12 nuits, des couples vont pouvoir mesurer la force et la faiblesse de leur amour. Et spécialement répondre à cette question: "suis-je avec la bonne personne?" “. Les candidats se retournèrent contre les producteurs, arguant qu'il ne s'agissait pas du tout d'une aventure enrichissante pour le couple, mais d'un travail. Pour se défendre, les producteurs opposèrent qu'il ne s'agissait que d'un jeu, d'ailleurs régulé par un "règlement" et pas par un contrat. Pour s'opposer à ces arguments, les avocats des plaignants sortirent l'Ile de la tentation de la sphère du divertissement et la ramenèrent vers la réalité, tout en gardant en ligne de mire l'horizon de la fiction. Ils l'emportèrent et l'arrêt note que le plaignant “ a pu parfois, pour satisfaire au concept de l'émission, être orienté dans l'analyse de sa conduite et répéter certaines scènes filmées". Par exemple, les producteurs, comme d'habiles metteurs en scène, ont parfois conditionné les réactions des candidats en leur donnant de fausses informations sur leur partenaire, ils leurs ont fait redire certaines répliques pour les rendre plus vivantes ou plus crédibles. Le participant n'est donc pas loin d'être un acteur.

Un tel exemple montre la difficulté à ne s'en tenir qu'au critère de la responsabilité énonciative. La frontière qui sépare le joueur du comédien est ténue: ne dit-on pas d'ailleurs des deux qu'ils jouent? Si l'on conçoit la communication, non comme une chambre d'enregistrement, mais comme un processus complexe, dont le conflit n'est pas absent, comme l'attestent les multiples procès qu'engendre la question du genre télévisuel, on voit qu'il faut changer à nouveau de paradigme et considérer que chaque programme et chaque genre est déchiré entre plusieurs mondes qui subsument les modes d'énonciation. D'où l'idée que les genres dépendent sémantiquement d'archigenres qui les contiennent et les sémantisent. Aux trois modes d'énonciation, je propose donc de substituer ces trois mondes:

a) le monde réel - la première question est de savoir si les signes font référence à des objets existants dans notre monde, ou s'ils font référence à de pures "chimères", des entités fictives. Notre monde est l'interprétant de nombreux genres, comme le documentaire ou le journal télévisé, ce qui ne veut pas dire que leurs signes soient transparents ni que les programmes restituent le monde. Cela ne préjuge pas non plus du modèle de la réalité mise en œuvre historiquement par ces genres; 
b) le monde fictif - la fiction, dont nous avons vu que le statut est variable selon la capacité des téléspectateurs à suspendre leur incrédulité;

c) le monde ludique - celui-ci se différencie des deux autres, d'une part, du fait que l'activité ludique est une activité au second degré ; qui obéit certes à des règles, comme la fiction, mais dont l'issue n'est pas donnée et dont la finalité n'est pas ancrée dans un Je-origine fictif, mais dans Je-origine réel.

$\mathrm{Au}$ " pour de vrai " de l'information, qui prend le monde comme référent, au "pour de faux" de la fiction, qui vise un univers mental, il faut donc ajouter un "pour de rire". Cette nouvelle tripartition est aussi une réponse à la critique qui m’a été adressée quant au déséquilibre entre l'action suggérée par le mode authentifiant, et la position statique des modes fictifs ou ludiques. En effet, c'est un processus logique qui génère les interprétants des documents: le signe est interprété par renvoi à un objet réel (la réalité) ou imaginaire (la fiction), ou il se prend lui-même comme objet, ce qui est toujours le cas du jeu, qui fonctionne en partie en circuit fermé et sous forme réflexive, obéissant aux règles qu'il s'est fixées.

Si le premier paradigme proposé dans cet article (ontologique) suppose la connaissance des genres, le deuxième (les modes d'énonciation) prend en compte la reconnaissance d'un certain nombre de critères qui amènent le téléspectateur à choisir plutôt tel interprétant que tel autre, les choses se compliquent encore un peu quand on replace l'interprétation dans le contexte de la communication télévisuelle. Le fonctionnement de la télé-réalité démontra que la question du genre ne se réduisait pas à la promesse ontologique ou à l'horizon d'attente inhérent à un genre, mais que, dans une perspective communicationnelle, l'opération la plus importante et, à la fois, la plus complexe à décrire, était l'attribution d'un nom à un programme. Cet acte de baptême, qui donne à un programme son identité engage, en effet, non pas l'invention du mot juste, celui que trouverait un onomaturge tout droit sorti du Cratyle de Platon, mais l'énonciation d'une promesse sur le bénéfice symbolique acquis à l'écoute du programme, une promesse pragmatique qui engage évidemment son énonciateur, producteur ou chaîne, mais aussi les savoirs et les croyances du téléspectateur. Car l'attribution d'un nom de genre à un programme donné n'est nullement une opération qui relève de la vérité ou d'un processus incontestable. Ce phénomène n'est d'ailleurs pas propre à la télévision.

Chacun garde en tête l'histoire de cette petite fille juive adoptée par des loups pendant la guerre pour retrouver ses parents (Survivre avec les loups; titre original: Misha: A 
Mémoire of the Holocaust Years). Elle fut d'abord présentée comme une autobiographie dans tous les médias sans que personne ne tique, et, quelques mois plus tard, l'imposture ou l'escroquerie fut dévoilée: il ne s'agissait que d'un roman, qui, du coup, perdait de son intérêt. Pour les nouveaux formats de la télévision, ces glissements sémantiques sont constants: Popstars (Version française et brésilienne de Pop Idol) a reçu des subsides réservés au documentaire ${ }^{2}$, au grand dam des réalisateurs, qui finalement plaidèrent devant le juge que Popstars "a pour principal objet de présenter au public l'entraînement, la formation et la progression, dans le domaine de la chanson, des personnes sélectionnées et de décrire un début de carrière effective, au sein des métiers du spectacle ", ajoutant que l'émission " comporte des éléments de scénario, une mise en scène et un montage ", qui sont des éléments propres à "l'œuvre télévisuelle". Des "docu-fictions" prétendent livrer une vérité historique. Chaque jour de nouveaux conflits d'attributions génériques surgissent. L'un des plus récents est celui de la "scripted reality".

Ces programmes sont fondés sur des faits divers. L'un d'entre eux - Le Jour où tout a basculé - était à l'origine précédé d'une présentation d'une avocate qui en authentifiait la source. L'aventure est jouée par des acteurs, ce qui la met clairement du côté de la fiction, en vertu du critère du Je-origine. Cependant, l'intervention des personnages, qui viennent face caméra expliquer leurs motivations, leurs sentiments ou leurs pensées, est un indice de factualisation empruntant aux diverses représentations du témoignage des reality shows, qui pèsent sur l'interprétation fictive. On ne s'étonne pas, dans ces conditions, que ce genre de programme ait fait récemment l'objet d'une discussion que le Conseil supérieur de l'Audiovisuel devra trancher. Du point de vue de la promesse faite aux téléspectateurs, il s'agit sans ambiguïté d'un programme renvoyant à la réalité. Pour les producteurs, au contraire, le recours à des scénaristes et à des comédiens prouve que ce sont des fictions. Cette dispute n'est pas une simple joute verbale sans importance car, si on range ces programmes du côté de la fiction, du même coup, la chaîne peut les comptabiliser dans les œuvres de création française et satisfaire plus facilement ses quotas à peu de frais.

${ }^{2}$ Ce programme racontait l'histoire de jeunes gens qui devaient former un groupe musical. Les réalisateurs se plaignirent qu'il ne s'agissait pas d'un documentaire - qui suppose que l'on filme ce qui existe déjà et non que l'on construise la réalité pour la caméra - que c'était plutôt une émission de variétés, mais le conseil d'état jugea le contraire. 


\section{Quel paradigme pour interpréter les genres télévisuels?}

$\mathrm{Au}$ terme de ce parcours, on peut répondre à la question posée par mon titre de la façon suivante.

Penser les genres en termes d'inférence - premier modèle - ; c'est décrire ce que pourrait être la réception des genres lorsqu'elle est fondée sur un savoir. C'est donc décrire une sorte de cadre idéal - qui n'est sans doute pas le plus proche de la réalité empirique mais qui pourrait relever de l'éducation aux médias puisqu'il s'agit de déterminer ce que l'on peu attendre d'un type générique en matière de codes audiovisuels. Le tableau des inférences produites par le spectateur en fonction des genres n'est valable que pour celui qui sait ou, tout simplement, qui prend en compte ce qu'il est permis d'attendre d'un genre (un peu à la façon de ce programme de la morale kantienne: “que m'est-il permis d'espérer?"). Cela permet ipso facto de contrecarrer certaines manipulations ou certains mensonges. Par exemple, pendant la retransmission soi-disant en direct de la révolution roumaine, le changements de caméra infirmaient le possibilité même du direct dans la mesure où celui-ci aurait supposé des moyens que insurgés ne pouvait avoir.

Penser le genre comme mode d'énonciation relève de la démarche analytique d'une sémio-narratologie. Cela suppose que la ligne de partage essentielle entre réalité et fiction passe par le sujet d'énonciation, et non par l'objet représenté ou raconté. Or il suffit d'être attentif aux discours sociaux comme aux récits des réceptions particulières pour constater que ce retournement copernicien supposé par la théorie de K. Hamburger est loin d'être entré dans les mœurs. Dès qu'un film prétend s'appuyer sur une histoire vraie, par exemple, les frontières deviennent floues aux yeux des spectateurs qui le verse souvent du côté de la réalité (comme le prouve la qualification de Valse avec Bachir en documentaire). De plus, ce paradigme ne peut être mise en œuvre que dans le cas de genres assez simples - comme Joles parle de "formes simples" -, pour lesquels il est possible de déterminer une responsabilité énonciative.

Or il n'est pas nécessaire d'avoir fait des études de narratologie pour recevoir un film. Et, même quand on sait ce qu'est un documentaire, on peut fort bien communiquer un téléfilm de fiction comme s'il était un documentaire (c'est le cas de maints "docu-fictions", de l'Odyssée de l'espèce à certains téléfilms historiques). La télévision est un processus communicationnel précisément parce qu'elle envoie ces objets, que sont les programmes dans un espace commun au producteur, au diffuseur, aux médiateurs et aux téléspectateurs, et que ces objets sont appropriés par chacun par une opération qu'on peut définir comme 
l'ancrage dans un monde. Contrairement à la description du genre en fonction des modes d'énonciation, qui supposent un savoir, cet ancrage dans un monde ne présuppose aucune vérité: il qualifie une opération d'attribution, qui est commune à tous les acteurs de la communication, mais dont le résultat diffère, selon qu'on est crédule, critique ou complaisant. Selon aussi l'image qu'on a du diffuseur qui envoie le programme et la capacité que l'on a à mettre en cause son autorité (comme nous l'apprend le faux JT belge).

Un même programme peut appartenir à des genres différents tout comme n'importe quel objet littéraire. Un talk show renvoie au monde réel, mais il peut aussi, comme joute verbale, être considéré comme un jeu. Il est néanmoins possible d'établir des règles constitutives de ce que sont et ne sont pas les mondes qui servent d'interprétants aux genres: ce qui suppose une réflexion approfondie sur les relations entre réalité et fiction, fiction et jeu. C'est au théoricien - sémioticien, philosophe - d'élaborer ses critères. De ce point de vue, toutes les propositions de sens méritent examen mais elles ne sont pas également admissibles. On a vu qu'accepter, par exemple, qu'un concours de chant soit assimilé à un documentaire n'est pas légitime si l'on s'accorde sur le fait qu'un documentaire doit rendre compte d'un état du monde qui le précède et qui n'est pas spécialement conçu pour être filmé. Selon la connaissance de ces règles, selon son attention, selon sa culture, le récepteur est plus ou moins enclin à valider les propositions de sens de la chaîne.

Une éducation à la télévision doit se situer à l'interface de l'émetteur et du récepteur. Tenant compte des propositions de sens des chaînes et de ce qu'il sait des règles constitutives des mondes, le sémiologue est le médiateur qui envisage la confiance que l'on peut leur faire, en gardant en tête cette double distinction que Roland Barthes assignait à ses Mythologies (1957): le "démontage sémiologique" et la "critique idéologique".

\section{Références}

BARTHES, Roland. Mythologies. Paris: Seuil, 1957.

ECO, Umberto, Six promenades dans les bois du roman. Paris: Grasset, 1996.

ECO, Umberto. TV: la transparence perdue. La Guerre du faux. Paris: Livre de poche, [1983] 1991.

GENETTE, Gérard. Fiction et diction. Paris: Seuil. 1991.

HAMBURGER, Käte. Logique des genres littéraires. Paris: Seuil, 1986.

HENRIOT, Jacques. Sous couleurs de jouer: la métaphore ludique. Paris: Corti, 1989. 
JOST, François. Un Monde à notre image: énonciation, cinéma, télévision. Paris: Méridiens Klincksieck, 1992.

JOST, François. Le Temps d'un regard. Paris-Montréal: Méridiens Klincksieck-Nuit Blanche, 1998a.

JOST, François. Quand y a-t-il énonciation télévisuelle? In: BOURDON, J. ; JOST, F. (Dir.).

Penser la télévision: actes du colloque de Cerisy. Paris: Nathan, 1998b.

SCHAEFFER, Jean-Marie. Qu'est-ce qu'une genre littéraire? Paris: Seuil, 1989.

SPERBER, Dan; WILSON, Deirdre. La pertinence: communication et cognition. Paris: Minuit, 1989.

\section{Qual o melhor paradigma para se interpretar os gêneros televisivos?}

\section{Resumo}

A quantidade de gêneros que caracterizam os programas televisivos é grande e cresce a cada dia. Como definir quais os interpretantes fundamentais que nos permitiriam pensar, não apenas nos gêneros do passado, mas também naqueles que estão por vir? É essa questão que busco responder, através de um modelo conceitual que evoluiu no decorrer dos anos, passando especificamente de uma caracterização de modo de enunciação, para uma caracterização de mundo. De modo a mundo, existe uma mudança de paradigma. Este artigo propõe, portanto, um retorno epistemológico a três jeitos de se enxergar os gêneros televisivos. Partindo de uma promessa que norteia as inferências e conviç̧ões dos telespectadores e que pode ser identificada, a princípio, a nível semiológico (vertente europeia da semiótica); em seguida pragmático, em decorrência do reconhecimento de um modo de enunciação que condiciona o valor de verdade de um programa; e, finalmente, pela definição dentro de um mundo, um modelo que explica o porquê das diferentes interpretações de gênero são um problema judicial, econômico e comunicacional.

\section{Palavras-chave}

Televisão. Gêneros televisivos. Interpretação. Enunciação. Promessa. Mundos. 


\title{
Which is the best paradigm to interpret the genres in television programming?
}

\begin{abstract}
The number of television genres, that is already big, grows more every day. How to define which are the fundamental interpretants that would allow us to think, not only about the genres of the past, but also about those to come? This is the question that I try to answer through a conceptual model that has evolved over the years particularly from a characterization of mode of enunciation, to a characterization of universe. From mode to universe there is a change of paradigm. This article proposes, therefore, an epstemological return to three different ways of seen the television genre. Starting from a promise that guide the viewers' interpretations and convictions, and that can be identified initially at a semiological level; then pragmatically, as a result of the recognition of a mode of enunciation that defines a TV program's value of truth; and finally by its definition inside a universe, a model that explains the reason why different genre interpretations constitute a judicial problem, as well as an economical and comunicational issue.
\end{abstract}

\section{Keywords}

Television. TV genres. Interpretation. Enunciation. Promise. Universes.

Recebido em 11/09/2015

Aceito em 15/12/2015 